\title{
Effective Properties of Cylinders in a Square Lattice
}

\author{
Brandon L. Good \\ Naval Surface Warfare Center \\ Carderock Division \\ West Bethesda, Maryland, USA
}

\begin{abstract}
Periodic subwavelength structures on a boundary can enhance the transmission through the boundary. While rigorous methods are accurate for designing these structures, recent methods could benefit from an improved effective medium approximation. In this work, a modified Rayleigh mixture formula is used to approximate the effective properties of periodic cylinders in a square lattice for transverse electromagnetic plane waves. The modified Rayleigh mixture formula outperforms the Maxwell Garnett formula where the height is greater than the diameter. A comparison is shown for completeness.
\end{abstract}

\section{INTRODUCTION}

Periodic subwavelength are useful for many microwave and optical applications. These application range from microwave impedance matching lenses [1] to optical resonators [2]. While numerous publications use periodic cylinders [1]-[6], additional exploration should be made into the implementation of these structures. In this work, an improved effective media equation will be formulated for periodic cylinders in a square lattice. This effective media equation will improve the efficiency of analytic and optimization methods of discrete (multilevel) and continuous (graded) cylindrical structures. This work will outline this new effective media formulation with increased accuracy.

\section{MaXWell-Garnett MiXture Formula}

While many effective mixture formulas exist, the MaxwellGarnett (MG) mixture formula is a direct analytic approximation for ellipsoid inclusions in a host medium [7]. The formula was derived from the scattering of a homogeneous dielectric sphere in the presence of an electrostatic electric field. For aligned ellipsoids the formula is:

$$
\varepsilon_{e f f, t}=\varepsilon_{h}+\varepsilon_{h} f \frac{\varepsilon_{i}-\varepsilon_{h}}{\varepsilon_{h}+(1-f) N_{x}\left(\varepsilon_{i}-\varepsilon_{h}\right)-R}
$$

Where $\epsilon_{e f f, z}$ is the effective permittivity for the mixture in the direction of the electric field, $\epsilon_{h}$ and $\epsilon_{i}$ are the permittivity of the host and inclusion, $f$ is the volume fraction of the sub wavelength structure, $N_{x}$ is the depolarization factor with regard to the direction of the electric field, and $R$ equals 0 for the standard MG formulation. A more complete descriptions of the MG mixture formula can be found in [7].

For a particular case where the ellipsoids are long $(h / d \geq$ 10) and in a square lattice, a 2D Rayleigh modification to the MG increases the accuracy of approximation. The 2D Rayleigh modification accounts for high interactions between cylindrical

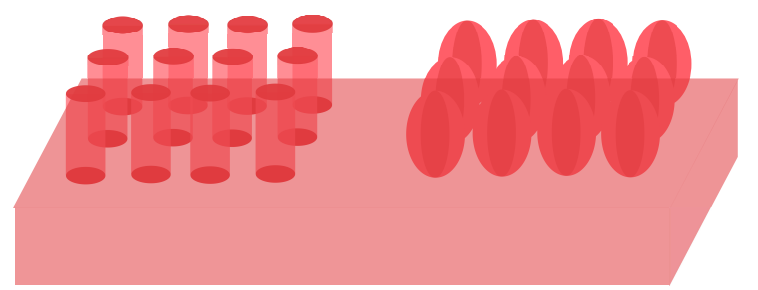

Fig. 1. Using prolate ellipsoids to model moth eye cylinders in a square lattice.

inclusions in a square lattice [8]. The MG formulation is modified by setting:

$$
R=\frac{\left(\varepsilon_{i}-\varepsilon_{h}\right)^{2}}{2\left(\varepsilon_{i}+\varepsilon_{h}\right)}\left(0.3048 f^{4}+0.0134 f^{8}\right)
$$

[8] has shown how the 2D Rayleigh formula outperforms the Maxwell-Garnett for this special case.

\section{EFFective Properties For a SquARe Lattic of CYLINDRICAL INCLUSIONS}

The approach derived here leverages the 2D Rayleigh and modifies ellipsoids geometry. The new effective media of cylindrical inclusions in a square lattice assumes that a prolate spheroids (ellipsoids) can be an approximation of a cylinder. Figure 1 shows the cylinder and the prolate spheroid. The challenge is appropriately matching the volume of the prolate spheroid to the cylinder. Since the height cannot change without maintaining the thickness of the effective properties, the increased volume of the ellipsoid must be put into the width $(a)$ of the ellipsoid. For the volume to maintained, the width of the ellipsoid is increased by $\sqrt{\frac{3}{2}}$.

Also hypothesized was the need for an additional scaling variable that would aim to account for the shape of the structure. Through trial and error, the square root of the inclusion permittivity seemed to be the best factor for increasing accuracy of the formulation. This would suggest that the cylinder exhibits a dependence on the optical path length. The cylinder shape depolarizes the effective properties along $\mathrm{z}$ axis. The final equation is shown below.

$$
\begin{gathered}
N_{t}=\text { prolatespheroid }\left(\frac{a}{c}=\frac{2 r}{h} \sqrt{\frac{3}{2} \epsilon_{i}}\right) \\
N_{z}=1-2 N_{t}
\end{gathered}
$$




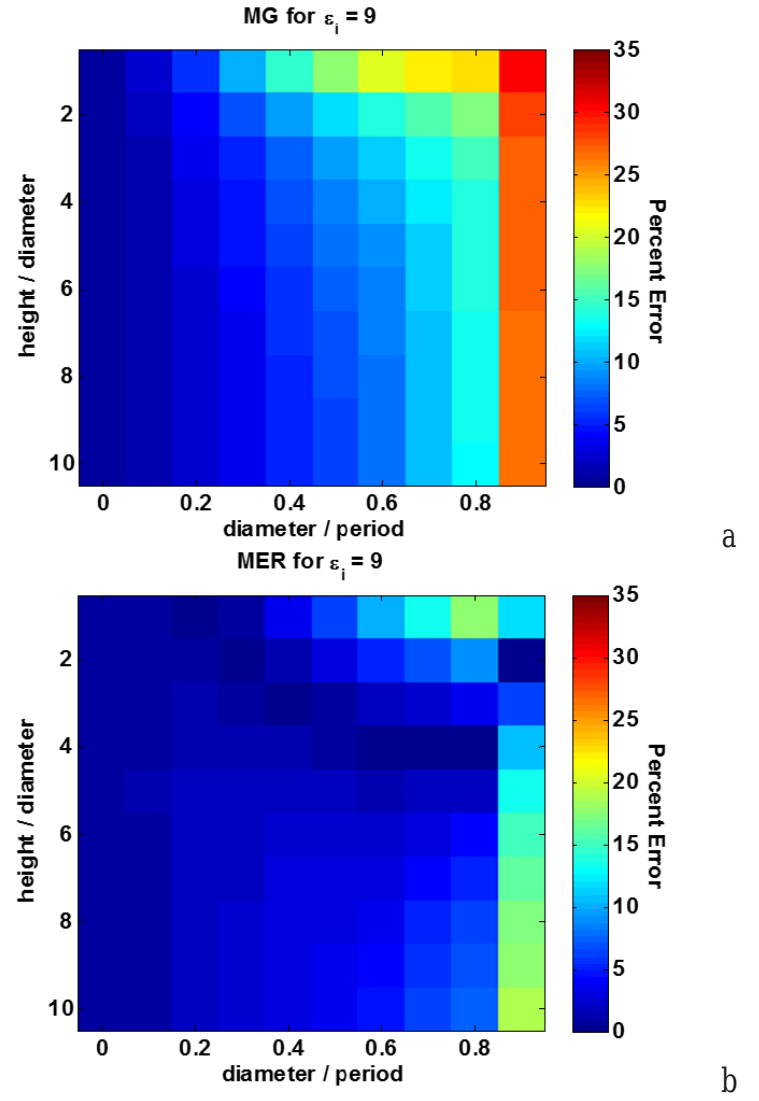

Fig. 2. Comparison between MG (a) and MER (b) for moth-eye structure with permittivity of 9

Where $N_{t}$ is the depolarization of the transverse axes ( $x$ and $y$ ) and $N_{z}$ is the depolarization of the $z$ axis. The depolarization formula for a prolate spheroid can be found in [7]. $N_{t}$ and $N_{z}$ are $N_{x}$ in equation 1 respectively. For the remainer of the work, this effective media approach will be called Modified Effective Rayleigh (MER). It should also be noted that the dyadic permittivity tensor will only work for oblique transverse electric plane waves.

\section{COMPARISON BETWEen MG AND MER}

To complete this analysis of the MER mixture formula for periodic cylinder in a square lattice, a comparison was done between the MER and MG approach. The comparison shown here consists of permittivity ranging from 2 to 25 , height to diameter $(h / d)$ ratio from 1 to 10 , and diameter to period $(d / p)$ ratio from 0 to 0.9 . The period was set to $\frac{\lambda}{20}$ to enable to be significantly sub-wavelength. The incident angle for this analysis is normal. The permittivity was derived using the method described in [4] with a rigorous coupled wave solution. The percent error was calculed by $\frac{\left|\epsilon_{\text {expected }}-\epsilon_{\text {predicted }}\right|}{\epsilon_{\text {expected }}}$. Figure 2 shows the error for MER and MG with an inclusion dielectric of 9. Every instance of the approximation of the MER has a lower error than the MG.

Figure 3 shows the mean and max values over the same $h / d$ and $d / p$ moth-eye profiles for a permittivity of 2 to

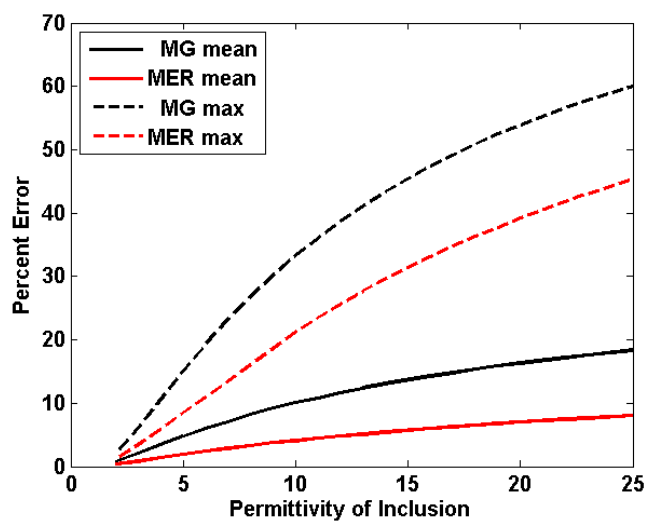

Fig. 3. Comparison between the mean and maximum error of MG (a) and MER (b) for moth-eye with permittivities from 2 to 25

25. From Figure 3, the mean and maximum error of the MER grows slower than MG. This would indicate that the MER is a suitable effective media formulation for periodic subwavelength cylinders in a square lattice.

\section{CONCLUSion}

A simple modified Rayleigh mixture approach was used to more accurately approximate the effective properties of periodic subwavelength cylinders in a square lattice. Future work will use this approximate to design multilevel and graded cylindrical designs.

\section{ACKNOWLEDGMENT}

The authors would like to thank the Office of Naval Research for supporting this work.

\section{REFERENCES}

[1] B. Good, P. Ransom, S. Simmons, A. Good, and M. Mirotznik, "Design of graded index flat lenses with integrated antireflective properties," Microwave and Millimeter wave Technology Letters, vol. 54, pp. 27742781, Dec. 2012.

[2] J. Caldwell, O. Glembocki, Y. Francescato, N. Sharac, V. Giannini, F. Bezares, J. Long, J. C. Owrutsky, I. Vurgaftman, J. Tischler, V. Wheeler, N. Bassim, L. Shirey, R. Kasica, and S. Maier, "Low-loss extreme subdiffraction photon confinement via silicon carbide localized surface phonon polariton reasonators," Nano Letters, vol. 13, pp. 3690-3697, 2013.

[3] M. Shokooh-Saremi and M. Mirsalehi, "Comparison of antireflective surfaces based on two-dimensional binary gratings and thin-film coatings," Applied Optics, July 2005.

[4] M. Mirotznik, B. Good, P. Ransom, D. Wikner, and J. Mait, "Iterative design of moth-eye antireflective surfaces at millimeter wave frequencies," Microw. Opt. Technol. Lett., vol. 58, pp. 561-568, Mar. 2010.

[5] - - "Broadband antireflective properties of inverse motheye surfaces," IEEE Trans. Antennas Propag., vol. 58, pp. 2969-2980, Sep. 2010.

[6] B. Good, S. Simmons, and M. Mirotznik, "Design of anti-reflection grading using magneto-dielectric materials," IEEE Trans. Antennas Propag., vol. PP, pp. 0-0, 2015.

[7] A. Sihvola, Electromagnetic Mixing Formulae and Applications. The Institution of Engineering and Technology, 1999.

[8] A. S. H. Wallen, H. Kettunen, Mixing Formulas and Plasmonic Composites. Springer Science + Business Media B. V., 2009, pp. 91-102. 